\title{
Open-source Architecture and Development Application of Door and Window Parameterized Design System
}

\author{
Shipei Cao ${ }^{\mathrm{a}}$, Yibing Qu ${ }^{\mathbf{b}^{*}}$, Xiangbo Ze ${ }^{\mathrm{c}}$, Zheng Liu ${ }^{\mathrm{d}}$, Kai Dong ${ }^{\mathrm{e}}$ \\ School of mechanical engineering,University of Jinan, Jinan City, Shandong Provice, China \\ a964754410@qq.com, ${ }^{\text {b }}$ \\ zexb_aop@qq.com, ${ }^{\mathrm{c}}$ 839352991@qq.com, ${ }^{\mathrm{d}}$ 778003129@qq.com, ${ }^{\mathrm{e}} 147378678 @ q q . c o m$
}

Keywords: doors and windows, parameterization, composition, open source CAD, custom, design

\begin{abstract}
To solve the problems of drawing complicated and repetitive labour when designing CAD drawings for windows and doors, a door and window parameterized design system based on open-source CAD was designed and developed. The system applies the concepts of composition and parameterization to the design of doors and windows. According to the principle of composition, the doors and windows are divided into units, the doors and windows are decomposed into several standard small units to form a standard unit library, and the unit of the unit library is called to be combined into a specific window type. After the window type design is completed, the user according to the specific window type custom parameters, parameterize the window type. Through practical use, the system can greatly improve the designer's design efficiency for new windows and save design time and costs.
\end{abstract}

\section{Introduction}

In the production of doors and windows industry, each project will contain a variety of window types, each window type will correspond to a number of sizes, and the window type between different projects is quite different. However, designers need to design a window of each size for these specific dimensions, which is a huge workload for designers Around the signing and implementation of product orders, rapid design of doors and windows is one of the most important influencing factors. ${ }^{[1]}$ and also increases the time cost of the enterprise. Our enterprises need for rapid design of window type, window type and reduce the repeated design process planning design dimensions necessary and relevant design information. ${ }^{[2]}$ In order to reduce the cost of the enterprise in the design and production, shorten the product design cycle, parametric design and manage-men $t$ of product repetitive features are imperative. At present, computer-aided design is widely used in various companies. In the continuous development of information technology, the application of computer-aided design technology has become more and more mature. ${ }^{[3]}$ When designing a window, the designer only needs to design various design elements and define the relevant attributes of various design elements. Then the elements are stored in the element library. When designing a specific window type, only the relevant elements need to be taken out from the element library, and the related parameters can be input to complete the design of a window CAD drawing.

\section{The Basic Principle of Open-source CAD Function Development}

\subsection{Two forms of open-source CAD extension development}

There are two forms of functional extension for open source CAD, one is to compile the source code of CAD, and to modify, extend and develop functional modules based on the $\mathrm{C}$ language to achieve the functions that they want to achieve; The form is to write a script program and use the scripting language such as Python to call the CAD API (Application Programming Interface) to write the function module that you want. The API can control the way CAD is run, eventually forming pyd formatted files and generating CAD extensions. 


\subsection{Introduction to open-source CAD development object}

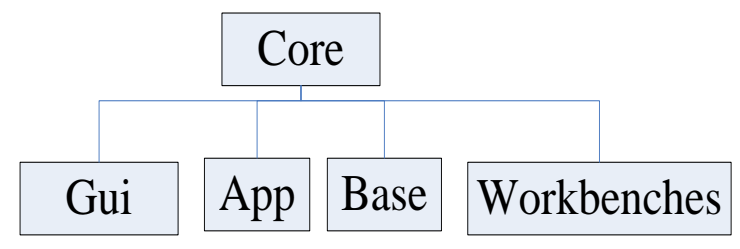

Figure 1 CAD Object.

As shown in Figure 1, the core objects of CAD, including Core, Gui, App, Workbenches, and some other auxiliary objects are not listed here. Core is the highest object in the CAD API and contains the core features of CAD. It groups the Base classes, and the main components of freecad core, spread over their App and Gui sides. Core components are programmed in $\mathrm{C}++$ but provide a broad Python API. The Base object provides some basic structure for other CAD objects, including most of the basic functions of CAD, such as printing different kinds of messages to the CAD report view or the terminal, handling the execution of Python code in CAD, and managing and saving user preferences settings. Management and Conversion of different Units. The App object is responsible for the management of other object attributes besides the Gui. Through the App module, you can manage document properties, obtain the attributes of objects such as sketches and three-dimensional drawings in the drawing, and add and change the properties of the objects through the App to control objects in the drawing area. The Gui object manages the graphical user interface and 3D view of the CAD. Some external commands can be registered in the CAD system structure through the GUi object to implement an external script to operate the CAD interface, and provide a possibility for the module to implement one or more workbenches. Workbenches objects are some of CAD's additional features and GUI tools, that is, some of the plugins written in C++ and Python for CAD. These plugins are also called modules or workbenches. Each workbench is a module written for some specialized functions in CAD.

\section{Development Methods and Key Technologies of Door and Window Parameterized Design System}

\subsection{Door and window parameterized design system development method}

This article mainly introduces the Python language, supplemented by open source CAD's macro recording function to extend the development of open source CAD. When the program is written, the code obtained by the macro recording function is used as a reference, and then edited and modified appropriately, and then added to the code of the application program. ${ }^{[4]}$ In this way, the secondary development of CAD by using the CAD macro recording function as an aid can not only greatly improve the programming efficiency but also make it easier for engineers to get started. However, this method cannot change the internal API of CAD, and is limited by the number and quality of APIs provided by CAD, but overall this method has more advantages than disadvantages.

\subsection{The development process of door and window parameterized design system}

As shown in Figure 2, the development process is illustrated as follows: First of all, in the open source CAD environment, according to the correct design steps, draw a CAD drawing with clear size and geometric shape. And within the interface of the two development, the user defines its variable parameters and the quantitative relationship between the parameters, and then stores the data and graphics into the database. When the graph is used again, the parameters previously defined are changed through the interface. The program can obtain and modify the relevant parameters by calling the written API objects, changing the geometric information and constraint information of the graphics, so that the whole process is completed. 


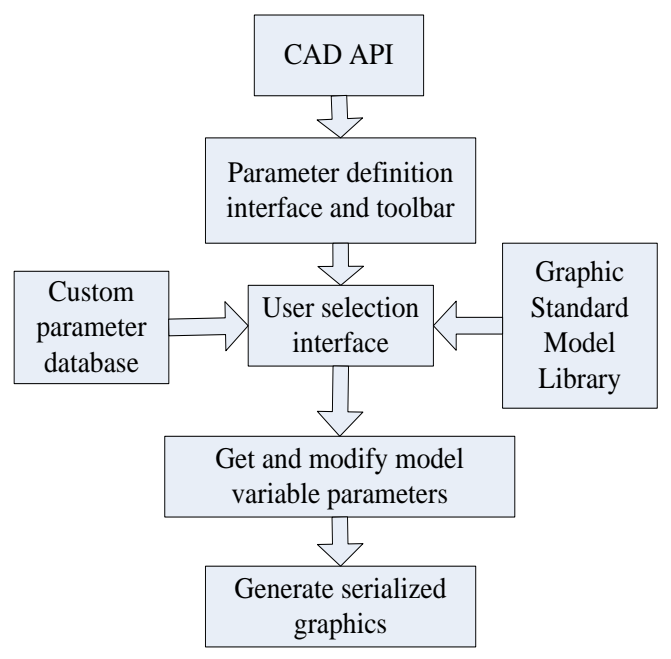

Figure 2 Development Flow Chart.

\subsection{Key Technology for the Development of Door and Window Parameterized Design System}

The following describes the key technologies in the functional extension development of open source CAD based on the Python2.7 programming environment through the parameterized design of doors and windows.

\subsubsection{Geometry unit determination}

The design of this system adopts the concept of combination. Combination is also called modularization. It uses the principle of decomposition and combination to decompose the functions of a class of products to form a general-purpose, relatively independent module system. Organize mass production, and then use the modules and other dedicated parts of the modular system to combine the new products needed.

Before the new geometry element is created, the window structure is decomposed using the idea of combination and decomposition to determine the geometric element to be created. The hardware, auxiliary materials, and glass that make up the entire window are not discussed here. As shown in the window structure diagram of Figure 3, the entire window can be decomposed into three parts: the outer frame, the inner fan, and the yarn fan, while the outer frame, the inner fan, and the yarn fan are composed of different unilateral shapes. After the entire window is decomposed, the geometrical unit is also determined. For the window-type structure, a new unilateral unit, a frame unit, an internal fan unit, and a yarn fan unit are newly established.

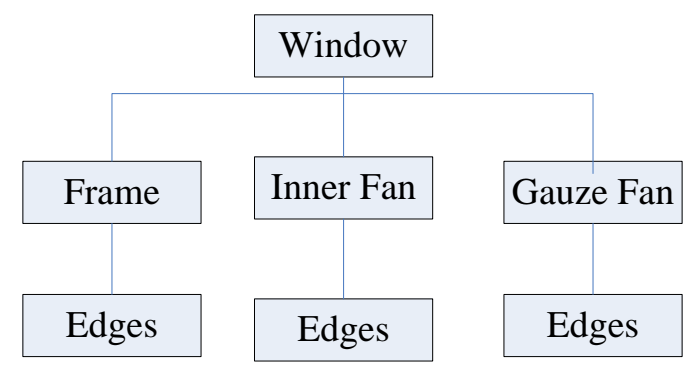

Figure 3 window structure.

\subsubsection{The representation of the geometry}

As shown in figure 4, if you want to express a graphic in open source CAD, you should express its geometric shape information and constraint relation information completely and accurately. The geometry information includes information such as the type of geometry element and the number of geometry elements. In Open Source CAD, the types of geometric elements mainly include five categories: line, Circle, ArcOfCircle, Ellipse, and Point. In CAD, these five basic elements are combined and they establish a constraint relationship and combine them into various complex 
graphics. The constraint relationship can be further divided into geometric constraints and engineering constraints. Geometric constraints are divided into structural constraints and dimensional constraints. Structural constraints mainly refer to the topological relationships between geometric elements, such as: parallel, vertical, etc.; Constraints represented by dimensions, such as: angles, distances, radius, etc.; engineering constraints refer to the constraint relationships between dimensions, such as adding numerical connections or equations between dimensions. ${ }^{[5]}$

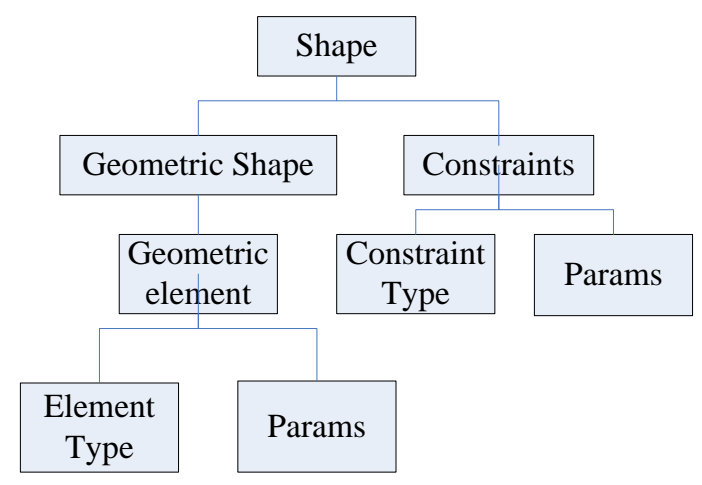

Figure 4 graphic structure

From the above analysis, we can use a binary array to represent its graphic information: shape = $\{\mathrm{GS}, \mathrm{C}\}$

Among:

- $\mathrm{GS}=\left\{\mathrm{Ge}_{1}, \mathrm{Ge}_{2}, \ldots, \mathrm{Ge}_{\mathrm{n}}\right\}$ is Geometric Shape. Express geometric information of graphics;

- $\mathrm{Ge}=\{$ GType,Params $\}$ is Geometric element. GType is the type of its geometry, and Params is the set of parameters it will pass;

- $\mathrm{C}=\{\mathrm{C} 1, \mathrm{C} 2, \ldots, \mathrm{Cn}\}$ is all Constraint information contained in the graph;

- $\mathrm{Cn}=\{$ CType,CParams $\}$, CType is its constraint type, and CParams is the parameter information needed for its constraint.

\subsubsection{Parameter setting}

When the new drawing is completed, as shown in Figure 5, the variables involved in the parameters, the correlation between the parameters, and the correlation function are added to the graphics.

\begin{tabular}{|c|c|c|c|c|c|}
\hline $\begin{array}{l}\text { Parameter Name } \\
1 \mathrm{H}\end{array}$ & & & 88 & Category & Re1-Formula \\
\hline $2 w$ & 1 & Stee1 Ind & 5 & $1 \mathrm{~W} 1$ & w/2+We1d Ind $* 2$ \\
\hline $\begin{array}{l}3 \mathrm{H} 1 \\
4 \mathrm{H} 2\end{array}$ & 2 & Weld Ind & 3 & $2 \mathrm{~W} 2$ & $\mathbb{W} / 2+$ We1d Ind $* 2$ \\
\hline 5 W1 & 3 & SWA & 10 & $3 \mathrm{H} 2$ & $\mathrm{H}-\mathrm{H} 1+$ We1d Ind $* 2$ \\
\hline
\end{tabular}

Figure 5 Setting parameters.

\subsubsection{User interface and main program design}

As shown in the figure 6, click on the window type in the interface. In the drawing area, the window type will be drawn and the custom parameters related to the window type will be listed in the following list. The values of these parameters will be changed in the drawing area. The associated size of the window map will be changed accordingly. 


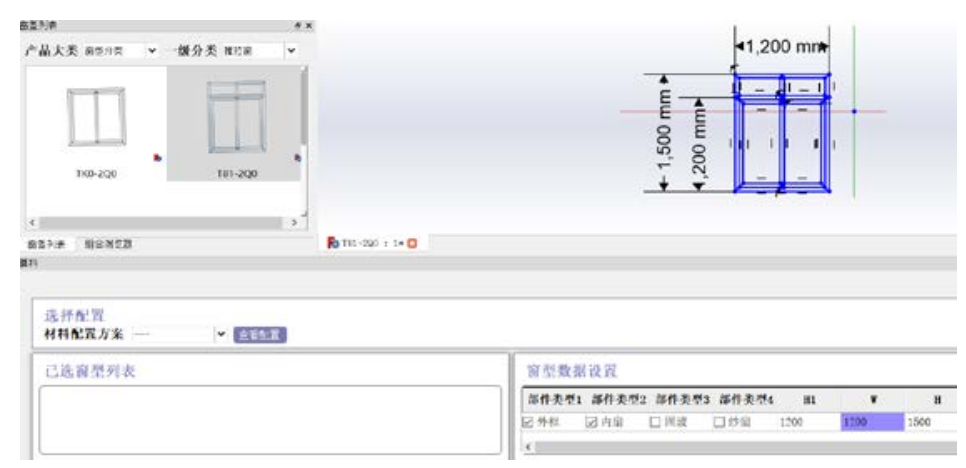

Figure 6 design interface.

The basic workflow is as follows:

- Establish a connection with open source CAD;

- Draw the window type through the window type

- Information stored in the database;

- Modify the set parameters;

- Re-draw the window based on the new size.

\section{Conclusion}

The key technologies and implementation ideas of the door and window parameterized design system are briefly discussed above. And through the application of the actual window design, this method is effective and feasible. Enterprises can use this idea and combine their own actual conditions and needs to define the required parameters for each window type, and establish their own window type library. This will undoubtedly greatly increase the efficiency of corporate door and window product design and shorten the development of window types. The cycle and the production cycle of orders have great practical significance.

\section{Acknowledgements}

This project is financially supported by the key research and development project of Shandong province (Grant No. 2017GGX203009), the project title is research on flexible production control system for pre-optimized multi-specification custom plastic doors and windows.

\section{References}

[1] Han Zhang. Plastic window assembly relationship automatic identification and parameterized design [D]. Jinan: University of Jinan, 2017.

[2] Kai Dong. Automatic recognition method of doors and windows fan area based on convex hull algorithm convex hull algorithm [A].Kai Dong. Proceedings of the 2017 6th International Conference on Measurement, Instrumentation and Automation (ICMIA 2017) [C]. Hong Kong: Atlantis Press, 2017.306-310.

[3] Jiming Wang. Computer Aided Design Technology Application Exploration [J]. Technology and Innovation. 2014(21):137,138,140.

[4] Chao Suo, YuXiang Li, Shuzhong Lin. Secondary Development of Parametric Design of Solid Works Based on VB Language[J]. Manufacturing Automation.2013, 35(8):137-140.

[5] Guoquan $\mathrm{Xu}$, Zhichao Huang. Parametric Design Secondary Development Based on SolidWorks [J]. Machinery Design and Research. 2007, 23(1): 69, 70, 76, 87. 